\title{
CURB-65 plus hypoalbuminemia: a new score system for prediction of the in-hospital mortality risk in patients with SARS-CoV-2 pneumonia
}

\author{
Alessandra Oliva1', Cristian Borrazzo', Maria Teresa Mascellino'1, Ambrogio Curtolo', \\ Dania Al Ismail' ${ }^{1}$, Francesca Cancelli ${ }^{1}$, Gioacchino Galardo ${ }^{2}$, Tommaso Bucci ${ }^{3}$, \\ Giancarlo Ceccarelli' ${ }^{1}$, Gabriella d'Ettorre1, Francesco Pugliese ${ }^{4}$, Claudio M. Mastroianni' \\ Mario Venditti ${ }^{1}$ \\ ${ }^{1}$ Department of Public Health and Infectious Diseases, Sapienza University of Rome, Rome Italy; \\ ${ }^{2}$ Medical Emergency Unit, Sapienza University of Rome, Policlinico Umberto I, Rome, Italy; \\ ${ }^{3}$ Department of General Surgery, Surgical Specialties and Organ Transplantation "Paride Stefanini", \\ Sapienza University of Rome, Rome, Italy; \\ ${ }^{4}$ Anesthesiology and Intensive Care group, Sapienza University of Rome, Policlinico Umberto I, Rome, Italy
}

Article received 12 June, 2021; accepted 27 July, 2021

\section{SUMMARY}

Introduction: There is the need of a simple but highly reliable score system for stratifying the risk of mortality and Intensive Care Unit (ICU) transfer in patients with SARS-CoV-2 pneumonia at the Emergency Room. Purpose: In this study, the ability of CURB-65, extended CURB-65, PSI and CALL scores and C-Reactive Protein (CRP) to predict intra-hospital mortality and ICU admission in patients with SARS-CoV-2 pneumonia were evaluated. Methods: During March-May 2020, a retrospective, single-center study including all consecutive adult patients with diagnosis of SARSCoV-2 pneumonia was conducted. Clinical, laboratory and radiological data as well as CURB-65, expanded CURB-65, PSI and CALL scores were calculated based on data recorded at hospital admission. Results: Overall, 224 patients with documented SARS-CoV-2 pneumonia were included in the study. As for intrahospital mortality $(24 / 224,11 \%)$, PSI performed better than all the other tested scores, which showed lower AUC values (AUC $=0.890$ for PSI versus $\mathrm{AUC}=0.885$, AUC $=0.858$ and $A U C=0.743$ for expanded CURB-65, CURB-65 and CALL scores, respectively). Of note, the addition of hypoalbuminemia to the CURB-65 score increased the prediction value of intra-hospital mortality (AUC=0.905). All the tested scores were less predictive for the need of ICU transfer $(26 / 224,12 \%)$, with the best AUC for extended CURB-65 score (AUC= 0.708). Conclusion: The addition of albumin level to the easy-to-calculate CURB-65 score at hospital admission is able to improve the quality of prediction of intra-hospital mortality in patients with SARS-CoV-2 pneumonia.

Keywords: CURB-65, extended CURB-65, hypoalbuminemia, PSI score, CALL score, CRP, mortality, ICU admission.
Corresponding author

Alessandra Oliva

E-mail: alessandra.oliva@uniroma1.it

\section{INTRODUCTION}

The novel coronavirus (now called SARS1 CoV-2) initially discovered in Wuhan, China, has spread all over the world causing a global pandemic [1-3]. All-cause mortality is approximately $10 \%$, as depicted by a recent meta-analysis conducted on 14.866 patients with SARS-CoV-2 infec- 
tion, with higher rate in patients admitted to Intensive Care Unit (ICU) or those developing Acute Respiratory Distress Syndrome (ARDS) [4-6].

During SARS-CoV-2 pneumonia a percentage of patients with mild symptoms might exhibit a sudden progression to severe or critical disease requiring intensive care $[7,8]$. In the latter case, the infection follows a characteristic pattern, with the development of respiratory failure and multi-organ dysfunction generally 7-12 days following symptom onset [9]. Therefore, there is the urgent need to consider simple and accurate predictors of adverse outcomes in order to early identify patients at risk of disease progression or death.

Multiple established risk scores have been used to assess the severity of community-acquired pneumonia (CAP) to improve management of CAP patients [10-12]. In detail, the first scoring system was the Pneumonia Severity Index (PSI), which consists of twenty clinical and laboratory parameters and assigns patients into 5 classes of risk [10]. However, although the PSI score is characterized by a high discriminatory power, it is complicated to calculate, especially at hospital admission. Therefore, the easier-to-calculate CURB-65 and extended CURB-65 scores have been suggested for CAP patients [11, 12].

In COVID-19 patients, several studies investigating demographic, laboratory and clinical parameters for outcomes prediction have been performed so far $[3,7,13-18]$. However, a very low-quality evidence and a striking heterogeneity among the populations have been also observed, making a definite conclusion very difficult. To be mentioned, the CALL score was recently developed to predict mortality whereas the role of admission serum C-reactive Protein (CRP) $>41.3 \mathrm{mg} / \mathrm{dL}$ as a predictor of adverse outcome in patients with SARS-CoV-2 infection has been highlighted by Luo and colleagues $[16,17]$.

Based on these premises, a simple but highly reliable score system is clearly needed for stratifying the risk of mortality and ICU admission in patients with SARS-CoV-2 infection at the Emergency Room. Therefore, in this study the ability of CURB-65, extended CURB-65, PSI and CALL scores and the value of CRP to predict intra-hospital mortality and ICU admission in patients with SARS-CoV-2 infection were evaluated. Furthermore, the single variables of each score sig- nificantly associated with the outcomes were also tested in addition to the scores in order to find the best combination able to predict mortality and need of ICU transfer.

\section{MATERIALS AND METHODS}

Study population

A retrospective single-center study including all consecutive adult patients with diagnosis of SARS-CoV-2 pneumonia and hospitalized at Azienda Policlinico Umberto I, Sapienza University of Rome during March-May 2020 was performed. The study was approved by the local Ethics Committee (ID Prot. 109/2020). SARS$\mathrm{CoV}-2$ diagnosis was based on nasopharyngeal swab positivity by using polymerase chain reaction (PCR) [19]. Definition of pneumonia or severe pneumonia was based on the WHO interim guidance and included clinical signs of pneumonia (fever, cough, dyspnea, fast breathing) with or without signs of severe pneumonia such as respiratory rate $>30$ breaths / $\mathrm{min}$, severe respiratory distress, or SpO2 $<90 \%$ on room air $[19,20]$.

For each subject, clinical, laboratory and radiological data at the admission and in-hospital mortality were collected and recorded anonymously in an electronic database. In particular, CURB-65, expanded CURB-65, PSI and the recently reported CALL scores were calculated based on data recorded at hospital admission [10, 11, 12, 16].

\section{Definitions of CURB-65, expanded CURB-65, PSI, CALL}

CURB-65 included Confusion, Blood Urea Nitrogen $(\mathrm{BUN})>7 \mathrm{mmol} / \mathrm{L}$, Respiratory rate $\geq 30 /$ min, Systolic Blood Pression (SBP) $<90 \mathrm{mmHg}$ or Diastolic Blood Pression (DBP) $\leq 60 \mathrm{mmHg}$, age $\geq 65$ years [11].

Expanded CURB-65 included CURB-65 plus the addition of lactate dehydrogenase $(\mathrm{LDH})>230 \mu / \mathrm{L}$, platelet $<100 \times 10^{9} / \mathrm{L}$, and albumin $<3.5 \mathrm{gr} / \mathrm{dL}$ [12]. PSI included age, sex, nursing home residency, neoplastic disease, liver disease, chronic heart failure, cerebrovascular disease, renal disease, altered mental status, respiratory rate $\geq 30 / \mathrm{min}$, systolic blood pressure $<90 \mathrm{mmHg}$, temperature $<35^{\circ} \mathrm{C}$ or $\geq 40 \mathrm{C}$, pulse $\geq 125 / \mathrm{min}$, arterial $\mathrm{pH}<7.35$, urea $\geq 11 \mathrm{mmol} / \mathrm{L}, \mathrm{Na}<130$, glucose $\geq 250 \mathrm{mg} / \mathrm{d}$, hematocrit $<30 \%$, $\mathrm{PaO} 2<60 \mathrm{mmHg}$ or $\mathrm{SaO} 2$ (air) $<90 \%$, pleural effusion [10]. 
CALL included Comorbidity, Age $>60$ years, Lymphocyte count $\leq 1 \times 10^{9} / \mathrm{L}, \mathrm{LDH}$ [16].

\section{Statistical analysis}

The data, unless otherwise stated, were given as medians with interquartile ranges (IQR, $25^{\text {th }}-75^{\text {th }}$ percentile), minimum and maximum values for continuous variables and as simple frequencies, proportions, and percentages for categorical variables.

Mann-Whitney was used for unpaired samples. Dichotomous variables were compared using Fisher's exact tests or chi-square test statistics. Receiver Operating Characteristic (ROC) curve analysis was used to calculate the optimal thresholds, sensitivities, and specificities for the 3 different scoring systems in study (CURB-65, CURB-65 extended, PSI and CALL). In order to assess the discriminatory power of the score for predict- ing outcomes, we compared the ROC curve of the CURB-65 score with those of the CURB-65 extended, PSI and CALL scores (or other combinations). Area under the ROC curve (AUC), 95\% Confidence Intervals (CIs) and p-values were considered the measure of the overall performance of each scoring system. The optimal threshold was defined as the value associated with the highest sum of sensitivity and specificity for each scoring systems (Youden index). Finally, we reported the sensitivity and specificity for each cut-off and then we were using the cut-offs to compare sensitivity and specificity of each score systems. The used cut-offs are described in Tables 1 and 2. The value of $41.3 \mathrm{mg} / \mathrm{L}$ for CRP was based on the results from Luo et al. [17].

P-value analyses were two-sided and a p-value of less than 0.05 was considered statistically significant. The Positive Predictive Values (PPV) and the

Table 1 - Prediction of in-hospital mortality compared to significant parameters.

\begin{tabular}{|l|c|c|c|c|c|c|c|}
\hline \multirow{2}{*}{ Markers } & \multirow{2}{*}{ Cut-off } & AUC & \multirow{2}{*}{ SE AUC } & $z$ & \multirow{2}{*}{ p-value } & \multicolumn{2}{c|}{ 95\% CI of AUC } \\
\cline { 5 - 9 } & & & & & LL & UL \\
\hline Expanded CURB-65 [12] & 2 & 0.885 & 0.030 & 13 & $<0.001$ & 0.827 & 0.942 \\
\hline CURB-65 [11] & 2 & 0.858 & 0.036 & 10.1 & $<0.001$ & 0.788 & 0.928 \\
\hline CRP $>$ 41.3 mg/L & $41.3 \mathrm{mg} / \mathrm{L}$ & 0.724 & 0.048 & 4.7 & $<0.001$ & 0.631 & 0.818 \\
\hline PSI [10] & 88 & 0.890 & 0.035 & 11.1 & $<0.001$ & 0.821 & 0.958 \\
\hline CALL [16] & 6 & 0.743 & 0.041 & 5.9 & $<0.001$ & 0.662 & 0.824 \\
\hline CURB-65 plus albumin $<3.5 \mathrm{~g} / \mathrm{dl}$ & 4 plus $<3.5 \mathrm{~g} / \mathrm{dl}$ & 0.905 & 0.030 & 12.8 & $<0.001$ & 0.822 & 0.938 \\
\hline CURB-65 plus CRP $>41.3 \mathrm{mg} / \mathrm{L}$ & 4 plus $>41.3 \mathrm{mg} / \mathrm{L}$ & 0.861 & 0.038 & 9.6 & $<0.001$ & 0.788 & 0.935 \\
\hline
\end{tabular}

Notes: Under non-parametric assumptions; null hypothesis: true area $=0.5$. *Statistical significance of AUC p-value $<0.05$. CRP: C-reactive protein; AUC: Area Under the Curve; SE: standard error; CI: confidence interval; LL: Lower limit; UL: upper limit.

Table 2 - ROC curve analysis for prediction of ICU transfer compared to significant parameters.

\begin{tabular}{|l|c|c|c|c|c|c|c|}
\hline \multirow{2}{*}{ Markers } & Cut-off & AUC & \multirow{2}{*}{ SE AUC } & $z$ & \multirow{2}{*}{ p-value } & 95\% CI of AUC \\
\cline { 5 - 9 } & & & & & LL & UL \\
\hline Expanded CURB-65 [12] & 3 & 0.708 & 0.048 & 4.361 & $<0.001$ & 0.614 & 0.801 \\
\hline CURB-65 [11] & 2 & 0.611 & 0.060 & 1.844 & 0.065 & 0.493 & 0.728 \\
\hline CRP $>41.3 \mathrm{mg} / \mathrm{L}$ & $41.3 \mathrm{mg} / \mathrm{L}$ & 0.637 & 0.048 & 2.871 & 0.004 & 0.544 & 0.731 \\
\hline PSI [10] & 88 & 0.658 & 0.057 & 2.752 & 0.006 & 0.545 & 0.770 \\
\hline CALL [16] & 6 & 0.648 & 0.053 & 2.825 & 0.087 & 0.545 & 0.751 \\
\hline CURB-65 plus albumin $<3.5 \mathrm{~g} / \mathrm{dl}$ & 4 plus $<3.5 \mathrm{~g} / \mathrm{dl}$ & 0.596 & 0.056 & 1.720 & 0.085 & 0.487 & 0.705 \\
\hline CURB65 plus CRP $>41.3 \mathrm{mg} / \mathrm{L}$ & 4 plus $>41.3 \mathrm{mg} / \mathrm{L}$ & 0.639 & 0.057 & 2.440 & 0.015 & 0.527 & 0.751 \\
\hline
\end{tabular}

Notes: Under non-parametric assumptions; null hypothesis: true area $=0.5$. ${ }^{*}$ Statistical significance of AUC p-value $<0.05$. AUC, Area under curve; SE, standard error; z, CI, confidence interval; LL, Lower limit; UL, upper limit. CRP: C-reactive protein; AUC: Area Under the Curve; SE: standard error; CI: confidence interval; LL: Lower limit; UL: upper limit. 
Table 3 - Demographics and baseline characteristics of patients with SARS-CoV-2 pneumonia.

\begin{tabular}{|c|c|c|c|c|c|c|c|c|c|c|}
\hline \multirow{2}{*}{ Parameters } & \multicolumn{3}{|c|}{$\begin{array}{c}\text { All patients } \\
(n=224)\end{array}$} & \multicolumn{3}{|c|}{$\begin{array}{c}\text { Survivors } \\
(n=200)\end{array}$} & \multicolumn{3}{|c|}{$\begin{array}{c}\text { Non-survivors } \\
(n=24)\end{array}$} & \multirow{2}{*}{ p-value } \\
\hline & $\begin{array}{l}\text { Median } \\
\text { (IQR) }\end{array}$ & $\begin{array}{l}\operatorname{Min} \text { to } \\
\max \end{array}$ & $n(\%)$ & $\begin{array}{l}\text { Median } \\
\text { (IQR) }\end{array}$ & $\begin{array}{l}\text { Min to } \\
\max \end{array}$ & $n(\%)$ & $\begin{array}{l}\text { Median } \\
\text { (IQR) }\end{array}$ & $\begin{array}{c}\text { Min to } \\
\max \end{array}$ & $n(\%)$ & \\
\hline Age $\geq 65 y$ & - & - & $102(46)$ & - & - & $82(41)$ & - & - & $20(83)$ & $<0.001$ \\
\hline $\begin{array}{l}\text { Systolic blood } \\
\text { pressure }<90 \mathrm{mmHg}\end{array}$ & - & - & $5(2)$ & - & - & $3(2)$ & - & - & $2(8)$ & 0.127 \\
\hline GCS $<15$ & - & - & $22(10)$ & - & - & $12(6)$ & - & - & $10(42)$ & 0.001 \\
\hline $\begin{array}{l}\text { Respiratory rate } \\
\geq 30 / \mathrm{min}\end{array}$ & - & - & $2(1)$ & - & - & $1(1)$ & - & - & $1(4)$ & 0.196 \\
\hline Platelet <100x109/L & - & - & $6(3)$ & - & - & $4(2)$ & - & - & $2(8)$ & 0.145 \\
\hline $\mathrm{BUN}>7 \mathrm{mmol} / \mathrm{L}$ & - & - & $62(28)$ & - & - & $44(22)$ & - & - & $18(75)$ & $<0.001$ \\
\hline albumin $<3.5 \mathrm{gr} / \mathrm{dL}$ & - & - & $68(30)$ & - & - & $49(25)$ & - & - & $19(79)$ & $<0.001$ \\
\hline $\mathrm{LDH}>230 \mu / \mathrm{L}$ & - & - & $166(74)$ & - & - & $146(73)$ & - & - & $20(83)$ & 0.130 \\
\hline $\mathrm{CRP}>41.3 \mathrm{mg} / \mathrm{L}$ & - & - & $107(48)$ & - & - & $89(45)$ & - & - & $23(96)$ & $<0.001$ \\
\hline PSI value [10] & $94(74-118)$ & $14-185$ & - & $64(49-84)$ & $21-179$ & - & $125(99-152)$ & $14-185$ & & $<0.001$ \\
\hline $\begin{array}{l}\text { Expanded } \\
\text { CURB-65 value [12] }\end{array}$ & $2(1-3)$ & $0-6$ & $70(31)$ & $1(1-2.5)$ & $0-5$ & - & $4(3-5)$ & $2-6$ & - & $<0.001$ \\
\hline CURB-65 value [11] & $1(0-2)$ & $0-4$ & $59(26)$ & $0(0-1)$ & $0-3$ & - & $2(2-3)$ & $0-4$ & - & $<0.001$ \\
\hline CALL value [16] & $10(8-15)$ & $7-17$ & - & $9(8-16)$ & $7-17$ & - & $11(10-11)$ & $9-13$ & - & $<0.001$ \\
\hline Death & - & - & $24(11)$ & - & - & - & - & - & $24(100)$ & - \\
\hline ICU transfer & - & - & $26(12)$ & - & - & $19(10)$ & - & - & $7(29)$ & 0.027 \\
\hline
\end{tabular}

Notes: GCS: Glasgow Coma Scale; BUN: Blood Urea Nitrogen; LDH: lactate dehydrogenase; CRP: C-reactive protein; ICU: Intensive care Unit

Negative Predictive Values (NPV) were calculated to evaluate the proportions of a true positive and negative results. All statistical analyses were performed with Statistical Package for Social Science (SPSS) version 22.

\section{RESULTS}

Over the study period, 224 patients with documented SARS-CoV-2 pneumonia diagnosed at the Emergency Room were included in the study. General baseline characteristics of the study population according to the parameters included in the tested scores are shown in Table 3. Age $\geq 65$-year was present in 102/224 subjects (46\%), high level of urea and hypoalbuminemia were detected in $62(28 \%)$ and 68 (30\%) subjects, respectively. Renal failure was present in 43 patients out of 224 (19\%) whereas diabetes was observed in $47 / 224$ subjects $(21 \%)$. A minor part of the studied population suffered from heart failure [21/224 (9\%)] and chronic obstructive pulmonary disease (COPD) was pres- ent in 34 out of 224 patients (15\%). The majority of patients showed high level of LDH (166/224, $74 \%$ ) whereas only few subjects presented with alteration in mental status, severe hypotension and low platelet count [22/224 (10\%), 5/ $224(2 \%)$, $6 / 224(3 \%)$, respectively], all parameters variably included in the tested scores. The intra-hospital mortality and need of ICU transfer were $11 \%$ $(24 / 224)$ and $12 \%(26 / 224)$, respectively. Among patients who died, 7 had been transferred to ICU whereas the other patients died for sudden worsening of the disease or before ICU transfer. A CRP value $>41.3 \mathrm{mg} / \mathrm{L}$ [17] was observed in $48 \%$ of subjects $(78 / 224)$. Overall, the median (IQR) values of PSI, CURB-65, extended CURB-65 and CALL scores were 94 (74-118), 1 (0-2), 2 (1-3) and 10 (8-15), respectively. Median length of hospitalization was 18 (11-26).

\section{Prediction of intra-hospital mortality}

The performance in predicting intra-hospital mortality by all the scoring systems is shown in Table 1 
and in Figure 1 by using ROC curves. Overall, the AUC of PSI score was superior to those observed for CURB-65 and extended CURB-65, which performed similarly (AUC $=0.890,95 \% \mathrm{CI}$, $0.821-0.958$ for PSI versus $\mathrm{AUC}=0.858,95 \% \mathrm{CI}$, $0.788-0.928$ and $\mathrm{AUC}=0.885,95 \% \mathrm{CI}, 0.827-0.942$ for CURB-65 and extended CURB-65, respectively). Conversely, the CALL score and the CRP $>41.3$ $\mathrm{mg} / \mathrm{L}$ showed lower AUC values $(\mathrm{AUC}=0.743$, 95\% CI, 0.662-0.824 and AUC $=0.724,95 \% \mathrm{CI}$, $0.631-0.818$, respectively). Interestingly, the annotation of hypoalbuminemia in addition to the CURB-65 score increased the prediction value of intra-hospital mortality, with an AUC $=0.905$ (95\% CI, 0.822-0.938), whereas adding the CRP value did not improve the performance of CURB-65 score (AUC $=0.861,95 \%$ CI, 0.788-0.935).

\section{Prediction of ICU transfer}

Overall, all the tested scores performed worse in predicting the need of ICU transfer, with the best AUC for extended CURB-65 score (AUC $=0.708$, $95 \%$ CI, 0.614-0.801). No adjunctive value was observed when adding albumin and CRP serum levels to CURB-65 score. The performance in predicting ICU admission by all the scoring systems is shown in Table 2 and in Figure 2.

Furthermore, we correlated the score data also

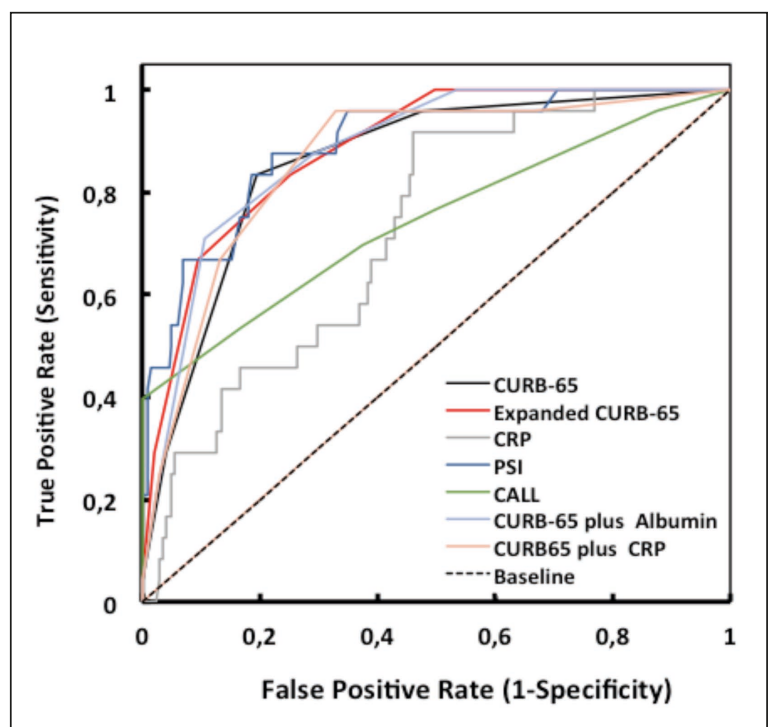

Figure 1 - ROC analysis using single and combined parameters for prediction of in-hospital mortality in patients with SARS-COV-2 pneumonia. with the length of hospitalization and we found that all the scores were not useful in predicting the outcome. In detail, the AUROCs were 0.519 (95\% CI 0.35-0.68), 0.491 (95\% CI 0.34-0.63), 0.598 (95\% CI 0.43-0.75), 0.503 (95\% CI 0.37-0.62), 0.572 (95\% CI $0.44-0.69), 0.428$ (95\% CI $0.27-0.58)$ and 0.569 (95\% CI 0.42-0.81) for CURB-65, extended CURB-65, CRP, PSI, CALL, CURB-65 plus albumin, CURB-65 plus CRP, respectively.

\section{DISCUSSION}

In this retrospective, single-center study the ability of different prognostic scores to predict intra-hospital mortality and ICU admission in patients with SARS-CoV-2 pneumonia was evaluated. We decided to include most of the scores commonly used at the Emergency Room for the management of CAP (i.e. PSI, CURB-65 and extended CURB65 ) and to include also COVID-19 specific items, such as the recently proposed CALL score and the CRP value [10-12, 16-17].

As for mortality, the PSI score performed best, with a median value belonging to class of risk IV, meaning an overall mortality of $9.3 \%$, very similar to what observed in our cohort of patients, whereas median CURB-65 and extended CURB65 accounted for low-to-moderate risk groups

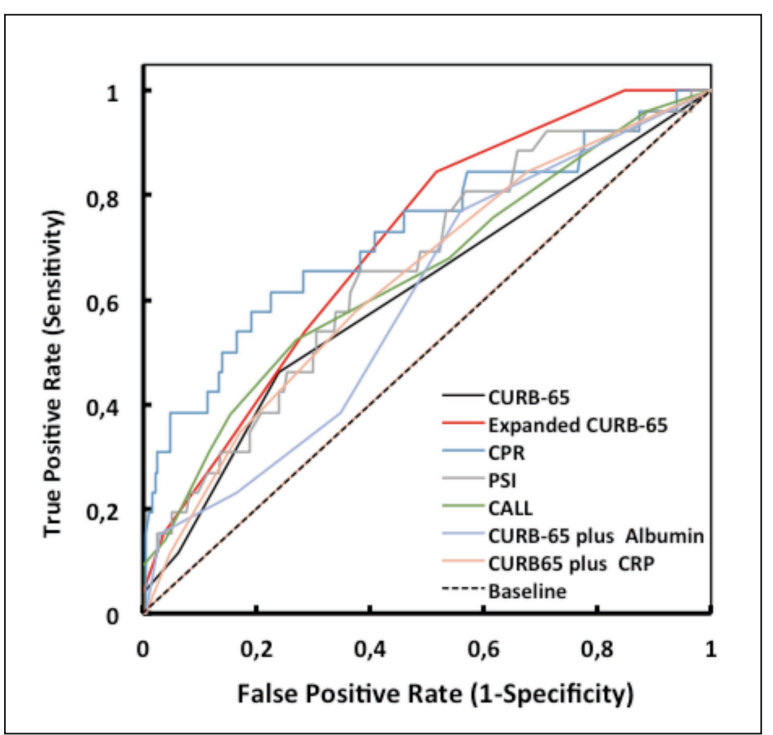

Figure 2 - ROC curve using single and combined scoring systems in the prediction of ICU transfer in patients with SARS-COV-2 pneumonia. 
(up to $6.8 \%$ of estimated mortality) [10-12]. This finding was then reflected by the higher AUC of PSI score than of CURB-65 and extended CURB65 scores, respectively, and suggests that CURB65 or extended CURB-65 alone may not be fully suitable for COVID-19. Overall, the tested scores seemed less predictive for the need of ICU transfer, at least in our population. However, given the paucity of study population, no definite data could be inferred and additional investigations are warranted.

Interestingly, both the CALL score and the CRP value, specifically designed for subjects with SARS-CoV-2 infection, were not able to predict the outcomes, rendering them not useful tools for discriminating patients at risk of death or ICU transfer, at least in our setting. In fact, the median value of CALL score was 10 (range 8-15), which corresponded to high risk of disease progression, significantly higher than that observed in our study [16].

The most interesting result of the present study was that expanding the CURB-65 score through the adjunct of hypoalbuminemia at hospital admission was able to increase the efficiency of predicting intra-hospital mortality in patients with SARS-CoV-2 pneumonia.

Several studies have identified a close correlation between hypoalbuminemia and mortality in CAP patients, whereas little is known as for SARS-CoV-2 pneumonia [21-27]. In fact, it has been recently demonstrated that an albumin level $<3.5 \mathrm{~g} / \mathrm{dL}$ was associated with worse outcome and coagulopathy [26-27]. Low albumin values could impair clinical outcomes even in children as shown in a recent study [28].

In order to understand the pivotal role of albumin in SARS-CoV-2 infection, we should be aware that serum albumin possesses anti-oxidant and anti-inflammatory properties and behaves as an inverse acute phase reactant during acute systemic inflammation [29-31]. Furthermore, stressed and inflamed cells increase the uptake of albumin from the circulation, thus highlighting a complex relationship between inflammation/infection and albumin level in the extracellular matrix [32-33]. Last but not least, albumin down-regulates the expression in the cells of the ACE2 receptors, which are known to be crucial in mediating and expanding SARS-CoV-2 infection [34-35]. Therefore, patients with reduced levels of albumin are predisposed to poor survival and, according to our report, the assessment of baseline albumin serum level at the Emergency Room in addition to the CURB-65 score may represent a crucial and easy-to-perform tool for the early identification of patients at higher mortality risk.

While several scores have been investigated and further validated for optimal for the management of CAP, for COVID-19 patients drawing a definite conclusion among the many proposed prognostic biomarkers and scores appears to be very difficult [13-17]. The CALL score and a risk score including several parameters calculated at hospital admission (age, sex, ethnicity, oxygen saturations, radiology, neutrophil count, CRP, albumin, creatinine, comorbidities) were recently developed to predict 28-day mortality and ICU admission $[9,16]$. Furthermore, a predictive model (named the HNC-LL score and based on neutrophil count, lymphocyte count, LDH level, CRP level and hypertension) was also developed and validated to enable early and accurate identification of patients with SARS-CoV-2 infection at a high risk of severe disease [36]. Finally, admission serum CRP at a cut-off value of $41.4 \mathrm{mg} / \mathrm{L}$ was shown to correlate well with disease severity and tended to be a good predictor of adverse outcome (i.e. mortality) in patients with SARS-CoV-2 pneumonia [17]. Taking into consideration the above mentioned scores, we decided to exclude from our analysis the score proposed by Galloway et al. since disease severity was radiologically assessed with adoption of chest radiograph examination that was replaced by chest CT in our patients population [9]. The HNC-LL score was highly predictive of severe disease, with the definition of severity including both subjects requiring intensive care and those with respiratory distress but not requiring ICU admission [36]. Even in this case, we did not include the HNC-LL score because we were mostly interested on mortality and ICU admission itself.

The present report has some clinical implications which should be highlighted. In fact, relying on a rapid and easy-to-perform instrument able to predict worse outcomes at hospital admission might represent a crucial step to early identify subjects with SARS-CoV-2 pneumonia at higher risk of intra-hospital mortality. Furthermore, the growing evidence that hypoalbuminemia plays a role in the survival rate of patients with COVID-19 might 
suggest its potential therapeutic use, especially taking into account the lack of a definite and specific (at least at the moment of writing) therapy.

The present study has several limitations. First, the retrospective and single-center nature of the study and the small number of included patients may have narrowed the interpretation of our results to a specific setting of population. Second, not all the published predictive scores in patients with SARS-CoV-2 pneumonia were included in our analysis. Third, all patients were treated according with the guidelines in force in Italy during the study period, which were changing over time. Therefore, we believe that correlating the different therapeutic strategies with the outcomes would have altered our data and, most importantly, our primary aim was to find a discriminative score at hospital admission, independently from the administered therapeutic regimens. Finally, we did not evaluate whether subjects at hospital admission presented with de-novo hypoalbuminemia or with pre-existing conditions associated with low-level of albumin. Such effect, however, would better reflect the features of patients with SARS-CoV-2 pneumonia at higher risk of worse outcomes.

In conclusion, our results suggested that the addition of the observation of a low albumin level to the easy-to-calculate and well-known CURB-65 score at hospital admission is able to improve the quality of prediction of intra-hospital mortality in patients with SARS-CoV-2 pneumonia. Further studies are needed to confirm this observation.

\section{Ethical approval}

The study was approved by the local Ethics Committee (ID Prot. 109/2020).

\section{Consent to participate and to publish}

Consent to participate and to publish were not obtained due to the retrospective nature of the study.

\section{Authors contributions}

Conceptualization, $\mathrm{AO}, \mathrm{MV}$; methodology, $\mathrm{AO}$, MV; data collection AO, FC, AC, DAI, TB, MTM, GG, GC, GdE; data analysis: $\mathrm{CB}, \mathrm{AO}$; writing-original draft preparation, $\mathrm{AO}, \mathrm{CB}$; writing-review and editing, CMM, FP, MV. All authors have read and agreed to the published version of the manuscript.

\section{Competing interests}

The authors declare no financial and non-financial competing interests.

\section{Availability of data and materials}

The data used to support the findings of this study are available from the corresponding author upon request.

\section{Fundings}

This research did not receive specific grants or fundings.

\section{Acknowledgements}

The authors thank the medical and nursing staff of Emergency and Infectious Diseases Departments for their contribution to patient's management.

\section{REFERENCES}

[1] Country \& technical guidance - coronavirus disease (COVID-19) [website]. Geneva: WorldHealth Organization; 2020 (https:/ /www.who.int/emergencies / diseases/novel-coronavirus-2019/technical-guidance). [2] Wu Z, McGoogan JM. Characteristics of and important lessons from the coronavirus disease 2019 (COVID-19) outbreak in China: summary of a report of 72314 cases from the Chinese Center for Disease Control and Prevention. JAMA. 2020; 323 (13), 1239-42.

[3] Grasselli G, Zangrillo A, Zanella A, et al. Baseline characteristics and outcomes of 1591 patients infected with SARSCoV-2 admitted to ICUs of the Lombardy region, Italy. JAMA. 323 (16): 1574-1581.

[4] Potere N, Valeriani E, Candeloro M, et al. Acute complications and mortality in hospitalized patients with coronavirus disease 2019: a systematic review and meta-analysis. Crit Care. 2020; 24 (1), 389. doi: 10.1186/ s13054-020-03022-1.

[5] Onder G, Rezza G, Brusaferro S. Case-fatality rate and characteristics of patients dying in relation to COVID-19 in Italy. JAMA. 2020; 323 (18), 1775-6 .

[6] Rajgor DD, Lee MH, Archuleta S, Bagdasarian N, Quek SC. The many estimates of the COVID-19 case fatality rate. Lancet Infect Dis. 2020; 20 (7), 776-7.

[7] Zhou F, Yu T, Du R, et al. Clinical course and risk factors for mortality of adult inpatients with COVID-19 in Wuhan, China: a retrospective cohort study. Lancet. 2020; 395 (10229), 1054-62.

[8] Li H, Liu S-M, Yu X-H, Tang S-L, Tang C-K. Coronavirus disease 2019 (COVID-19): current status and future perspectives. Int J Antimicrob Agents. 2020; 55, 105951.

[9] Galloway JB, Norton S, Barker RD, et al. A clinical risk score to identify patients with COVID-19 at high 
risk of critical care admission or death: An observational cohort study. J Infect. 2020; 81 (2), 282-288.

[10] Fine, M.J. Auble TE, Yealy DM et al. A prediction rule to identify low risk patients with community-acquired pneumonia. N Engl J Med. 1997; 336, 243-50.

[11] Lim, W.S. van der Eerden MM, Laing R et al. Defining community acquired pneumonia severity on presentation to hospital: an international derivation and validation study. Thorax. 2003; 58, 377-82.

[12] Liu J, Xu F, Zhou H, et al. Expanded CURB-65: a new score system predicts severity of community-acquired pneumonia with superior efficiency. Sci Rep. 2016; 18; 6:22911. doi: 10.1038/srep22911.

[13] Wu C, Chen X, Cai Y, et al. Risk factors associated with acute respiratory distress syndrome and death in patients with coronavirus disease 2019 pneumonia in Wuhan, China. JAMA 2020; Intern Med. e200994.

[14] Weiss P, Murdoch DR. Clinical course and mortality risk of severe COVID-19. Lancet 2020; 395, 1014-5.

[15] Liang W, Liang H, Ou L, et al. China Medical Treatment Expert Group for COVID-19. Development and Validation of a Clinical Risk Score to Predict the Occurrence of Critical Illness in Hospitalized Patients With COVID-19. JAMA Intern Med. 2020; 180 (8), 1-9.

[16] Dong J, Dawei Z, Jing X, et al. Prediction for Progression Risk in Patients with COVID-19 Pneumonia: the CALL Score. Clin Infect Dis. 2020; Apr 9; ciaa414. doi: $10.1093 /$ cid/ciaa414.

[17] Luo X, Zhou W, Yan X, et al. Prognostic value of C-reactive protein in patients with COVID-19. Clin Infect Dis. 2020; May 23; ciaa641. doi: 10.1093/cid/ciaa641. [18] Curtolo A, Oliva A, Volpicelli L et al. Monocyte absolute count as a preliminary tool to distinguish between SARS-CoV-2 and influenza A/B infections in patients requiring hospitalization. Infez Med. 2020; 28 (4), 534-8.

[19] World Health Organization. Clinical management of COVID-19, interim guidance 27 May 2020. https:/ / www.who.int/publications/i/item/clinical-management-of-covid-19.

[20] IMAI District Clinician Manual. Hospital care for adolescents and adults. Geneva: World Health Organization; 2020. https://apps.who.int/iris/bitstream/ handle/10665/77751/9789241548290_Vol2_eng.pdf?sequence, accessed 13 May 2020.

[21] Viasus D, Garcia-Vidal C, Simonetti A, et al. Prognostic value of serum albumin levels in hospitalized adults with community-acquired pneumonia. I Infect. 2013; 66, 415-23.

[22] Miyazaki H, Nagata N, Akagi T, et al. Comprehensive analysis of prognostic factors in hospitalized patients with pneumonia occurring outside hospital: serum albumin is not less important than pneumonia severity assessment scale. J Infect Chemother. 2018; 24, 602-9.

[23] Hatipoğlu U, Wells BJ, Chagin K, Joshi D, Milinovich A, Rothberg MB. Predicting 30-day all-cause readmission risk for subjects admitted with pneumonia at the point of care. Respir Care. 2018; 63, 43-49.

[24] Lee JH, Kim J, Kim K, et al. Albumin and C-reactive protein have prognostic significance in patients with community-acquired pneumonia. J Crit Care. 2011; 26, 287-94. [25] Xing Q, Dong X, Ren Y, et al. Liver Chemistries in Patients with COVID-19 Who Discharged alive or Died: A Meta-analysis. Hepatol Commun. 2020; ul 21;10.1002/ hep4.1585. doi: 10.1002/hep4.1585. Online ahead of print. [26] Violi F, Cangemi R, Romiti GF, et al. Is Albumin predictor of mortality in COVID-19? Antioxid Redox Signal. 2021; 35 (2), 139-42.

[27] Violi F, Ceccarelli G, Cangemi R, et al. Hypoalbuminemia, coagulopathy, and vascular disease in COVID-19. Circ Res. 2020; 127 (3), 400-1.

[28] Loffredo L, Campana A, Olivini N et al. Hypoalbuminemia and clinical adverse events in children with COVID-19. J Med Virol. 2021; 93 (5), 2611-13.

[29] Mani Mishra P, Uversky VN, Nandi CK. Serum albumin-mediated strategy for the effective targeting of SARS-CoV-2. Med Hypotheses. 2020; 24; 140: 109790. doi: 10.1016/j.mehy.2020.109790.

[30] Ferrer R, Mateu X, Maseda E, et al. Non-oncotic properties of albumin. A multidisciplinary vision about the implications for critically ill patients. Expert Rev Clin Pharmacol. 2018; 11, 125-37.

[31] Taverna M, Marie AL, Mira JP, Guidet B. Specific antioxidant properties of human serum albumin. Ann Intensive Care. 2013; 15 (3), 4.

[32] Finicle B.T., Jayashankar V., Edinger A.L. Nutrient scavenging in cancer. Nat Rev Cancer. 2018; 18, 619-33.

[33] Dirajlal-Fargo S, Kulkarni M, Bowman E, et al. Serum Albumin Is Associated With Higher Inflammation and Carotid Atherosclerosis in Treated Human Immunodeficiency Virus Infection. Open Forum Infect Dis. 2018; 5 (11): ofy291. doi: 10.1093/ofid/ofy291. eCollection.

[34] Liu BC, Gao J, Li Q, Xu LM. Albumin caused the increasing production of angiotensin II due to the dysregulation of ACE/ACE2 expression in HK2 cells. Clin Chim Acta. 2009; 403 (1-2), 23-30.

[35] Delgado-Ron JA. The potential use of albumin in COVID-19 patients. Response to Preventing a covid-19 pandemic. BMJ 2020; 368:m810. https: / / www.bmj.com/ content/368/bmj.m810/rr-41.

[36] Xiao L, Zhang W, Gong M, et al. Development and validation of the HNC-LL score for predicting the severity of coronavirus disease 2019. EBioMedicine. 2020; Jul; 57, 102880. doi: 10.1016/j.ebiom.2020.102880. Epub $2020 \mathrm{Jul} 7$. 